\title{
Using MPPCs for T2K Fine Grain Detector
}

\author{
Fabrice Retière ${ }^{1 *}$ \\ TRIUMF \\ 4004 WestBrook Mall \\ Vancouver BC V6T $2 A 3$ \\ Canada \\ fretiere@triumf.ca
}

Multi-Pixel Photon Counters from Hamamatsu Photonics will be used to read out the 8448 scintillator bars of T2K Near Detector Fine Grain Detector (FGD). The MPPCs are coupled to wavelength shifting fibers that are mirrored on the other side and go through a hole extruded in the middle of the $0.96 \times 0.96 \times 200 \mathrm{~cm}^{3}$ bars. We show that the MPPC performance meets the FGD specifications for dark noise rate, gain, gain fluctuation, and quantum efficiency despite fairly large cross-talk and after-pulsing. Beam test measurements show that minimum ionizing particles produce at least 10 photo-electrons when going through a FGD bar at a bias voltage where the after-pulsing probability is less than $10 \%$. After-pulsing has been found to be the main limitations of MPPCs as it rises quickly with bias voltage and exhibits two time constant of roughly 20 and 100 ns. MPPCs are read out by the newly designed AFTER ASIC. It is a 72 channel waveform shaper/digitizer, which is operated with a 120 ns peaking time and a $50 \mathrm{MHz}$ sampling frequency; the ASIC provides 10 microsecond continuous sampling. We show that the AFTER ASIC coupled to a MPPC provides very good energy resolution and about 3.5 ns timing resolution for MIP, while providing an efficient mean of identifying $\pi^{+}$ stopping in the scintillator bars by detecting the subsequent emission of Michel positrons.

International workshop on new photon-detectors PD07

Kobe University, Kobe, Japan

27-29 June, 2007

\footnotetext{
$1 \quad$ Speaker

* On behalf of the T2K ND280 FGD group: University of British Columbia, Kyoto University, University of Regina, TRIUMF, University of Victoria
} 


\section{Introduction}

The Fine Grain Detector (FGD) is an element of the T2K near detector located at $280 \mathrm{~m}$ from the neutrino producing target [1]. The near detector is designed to measure neutrino interactions close to the production target in order to obtain a large statistical sample. The FGD weighs almost one ton so that one neutrino interaction is expected per spill at nominal beam luminosity. It is built with scintillator bars alternating in the $\mathrm{X}$ and $\mathrm{Y}$ directions perpendicular to the beam. Particles emerging from the neutrino interactions generate scintillation light in the bars, which is recorded and used to reconstruct their trajectories. The neutrino vertex can then be identified within the FGD and some particle identification can be achieved by measuring particle energy loss. Further identification and momentum reconstruction is achieved in the Time Projection Chambers surrounding the FGD. In addition, positive pions can be identified if they stop within a FGD bar by detecting the Michel positrons following their decay. Protons stopping in the FGD are identified by their large energy loss. Neutrino interactions that lead to the production of neutral pions can be identified by associating charged particle signals recorded in the FGD with photons reconstructed in the electromagnetic calorimeter (ECAL). In order to reject background when combining the ECAL and FGD, a coincidence window will be used, which requires a timing resolution better than 5 ns per FGD bar.

The FGD consists of 2 separate detectors, one fully active, and one half filled with water, each $30 \mathrm{~cm}$ deep along the neutrino beam direction. The fully active FGD is segmented in $15 \mathrm{X}$ Y layers. Each layer direction is a stack of 192 bars, 2 meter long, and 0.96 by $0.96 \mathrm{~cm}^{2}$ across. The FGD containing water is used for understanding the difference in cross-section between scintillator and water, which Super-Kamiokande relies on for measuring neutrino interactions at the far end, $295 \mathrm{~km}$ away from the target. The water-scintillator FGD is made of $7 \mathrm{X}-\mathrm{Y}$ scintillator layers and 6 water layers. The total number of channels for both FGDs is 8448.

The scintillator bars will be read out by wavelength shifting fibers coupled to Hamamatsu Multi-Pixel Photon Counters (MPPC)[2]. The signal from each MPPC is readout by the AFTER ASIC designed to read out T2K Time Projection Chambers. It couples a preamplifier-shaper with a 511 cell Switch Capacitor Array (SCA). Using the AFTER ASIC allows to achieve the required timing resolution, while continuously sampling the signal for $10 \mu \mathrm{s}$, hence providing a very efficient detection of the Michel Positron. The read out system of the FGD combining MPPCs with the AFTER ASIC will be described in section 2.

The MPPC requirements are total number of pixels larger than 200, dark noise count rate < $1 \mathrm{MHz}$, cross-talk $<20 \%$, after-pulsing $<10 \%$ /pixel, gain larger than 50,000 and quantum efficiency equivalent or better than PMTs at the wavelength shifting fiber emission wavelength. We will verify that MPPCs meet such specifications in section 3. These requirements are derived from the FGD physics requirements: Minimum Ionizing Particle (MIP) reconstruction efficiency, timing and energy resolution, and dynamic range requirement for measuring stopping protons. 


\section{Reading out MPPCs with the AFTER ASIC}

The MPPC readout is designed to fulfill the FGD timing resolution requirements, while allowing the detection of Michel positrons from $\pi^{+}$decay following the $2.2 \mu$ s muon decay constant. The beam spill is $5 \mu$ s long, which requires the electronics to be alive for $10 \mu$ s in order to encompass the spill width and provide two muon lifetimes. The AFTER ASIC can be clocked at $50 \mathrm{MHz}$ and has 511 capacitive cells, which translates to $10.220 \mu \mathrm{s}$.

The timing resolution requirement is 3 ns per neutrino interaction, which corresponds to about 5 ns resolution per bars as multiple bars are combined. The MPPC signal rises very quickly ( $<1 \mathrm{~ns}$ ) and decay with a 8.75 ns time constant, which looks like a delta function at the input of the ASIC. The preamplifier-shaper streches the MPPC signal, the rise time being 120 ns in the short shaping configuration. Simulations show that a 3 ns timing resolution can be achieved when reading out a wavelength shifting fiber (7 ns decay constant) coupled to a MPPC with $50 \mathrm{MHz}$ sampling frequency and $120 \mathrm{~ns}$ preamp-shaper rise time, by fitting the waveforms.

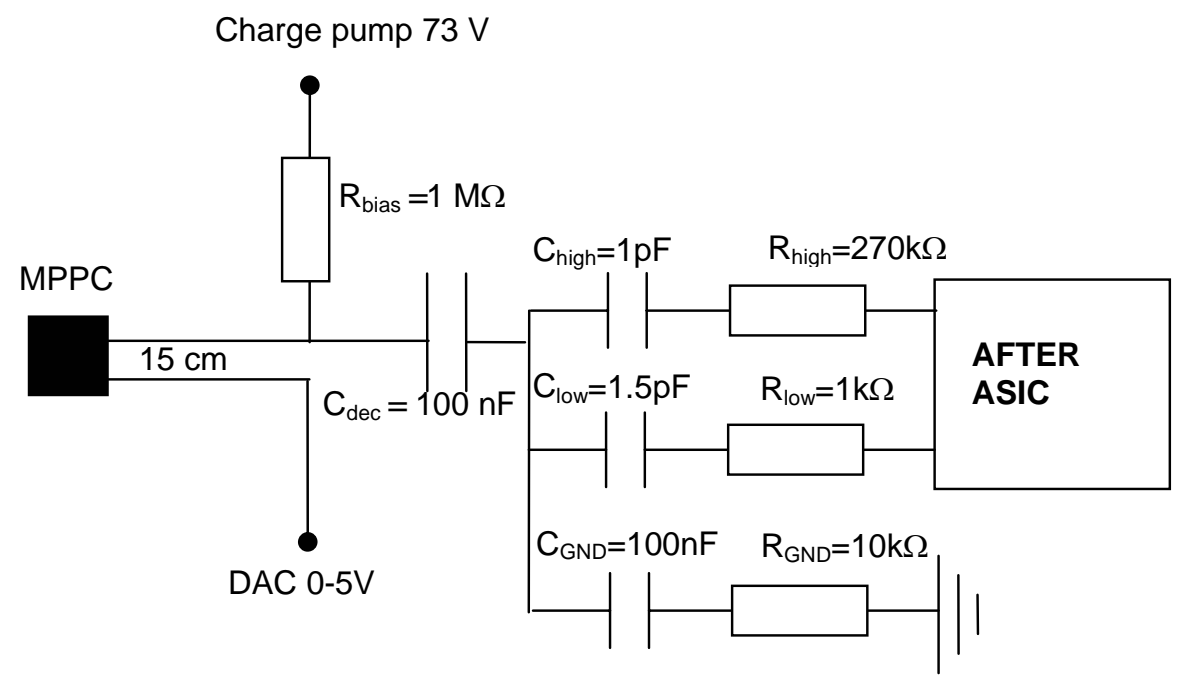

Figure 1. Schematics of the MPPC - AFTER ASIC coupling

The readout system must also fulfill the dynamic range requirement. The maximum charge at the output of the MPPC is the total number of pixel times the gain, which amount to $32 \mathrm{pC}$ with 400 pixels and the typical gain of $5 \times 10^{5}$. The maximum input charge of the AFTER chip is $600 \mathrm{fC}$, hence the signal must be attenuated. The lower bound of the dynamic range is given by the noise of the amplifier. In order to calibrate the MPPC gain, single pixel avalanche must be visible, hence the electronics noise must be less than 0.2 times the gain, which corresponds to $16 \mathrm{fC}$, or 100,000 e-. The noise of the AFTER chip is on the order of 2,000-3,000 electrons equivalent noise charge, which means that the signal must be attenuated by 30-50 in order to set the electronics noise to 0.2 equivalent photo-electron. However, given that the AFTER chip dynamic range is $600 \mathrm{fC}$, the maximum number of pixel avalanches that it can accept before saturating is then 225-375, which is less than the maximum number of pixels. Thus, for every MPPC, a second branch is fed into the ASIC with an attenuation of about 10. 
The AFTER chip signal to noise performance are optimum when the input capacitance is less than $30 \mathrm{pF}$. Since the total capacitance of the MPPC is $35 \mathrm{pF}$, it is advantageous to achieve the desired attenuation by using capacitor ranging between 1 and $20 \mathrm{pF}$. The schematics of the coupling between the MPPC and the AFTER ASIC is shown in Figure 1. The 1 and $1.5 \mathrm{pF}$ capacitors define the ASIC input capacitance. The desired attenuation is achieved by setting the value of the $R_{\text {High }}$, $R_{\text {Low }}$ and $R_{G N D}$ resistors. One drawback of using capacitors is that the line impedance cannot be exactly adjusted hence reflections are unavoidable. Given the shortness of the cables between the MPPC and the ASIC (about $15 \mathrm{~cm}$ ), and the long shaping time, the effect of reflexions is limited. The $1 \mathrm{M} \Omega$ resistance in series with the power supply ensures that the current never go above $100 \mu \mathrm{A}$ even if the MPPC shorts to ground. The $70 \mathrm{~V}$ bias, which is generated on the front end board by a charge pump circuit, delivers enough current to continue running even if MPPCs are shorted.
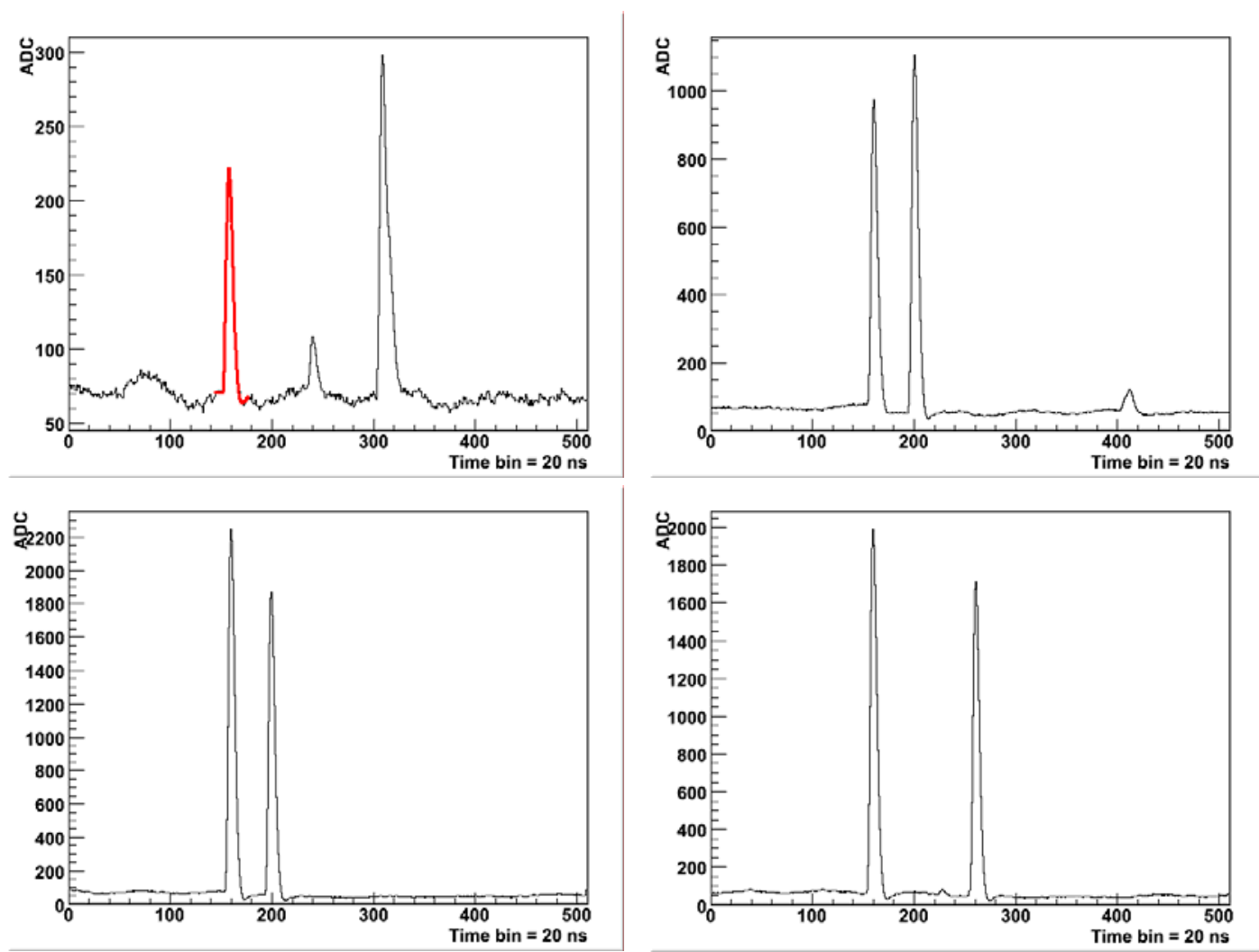

Figure 2. Typical waveforms obtained with different laser settings. Upper left, low light level (5 PE average), laser beam $3 \mu$ s apart. Upper right, 20 PE average light level, laser beams 800 ns apart. Lower left, 60 PE average light, laser beam 800 ns apart. Lower right, 60 PE average light, laser beams $3 \mu \mathrm{s}$ apart. The small pulses are single pixel avalanches due to dark noise.

A test setup has been built to investigate the performance of the MPPC coupled to the AFTER chip. The ASIC test board was designed for the TPC. A daughter board was built in order to bias the MPPC and split the signal between the high and low attenuation channels. Typical waveforms are shown in Figure 2. In this test setup, a $405 \mathrm{~nm}$ laser flashed the MPPC 
either directly or through a wavelength shifting fiber. Two laser pulses are fired within the integration window varying the laser light level and the delay between the first and second laser pulse.

The double laser pulse setup is used to measure the timing resolution and study the recovery of the MPPC bias after a large pulse. The timing resolution was found to be better than 5 ns when flashing a wavelength shifting fiber and when more than 10 photo-electrons are detected, which fulfills the requirements. Recovery of the MPPC bias depends on the details of the readout and bias circuit. The drawback of using a large resistance in series with the bias is that the MPPC bias recovery time constant is very long through this branch, typically $35 \mu \mathrm{s}$ (35 $\mathrm{pF}$ MPPC total capacitance times $1 \mathrm{M} \Omega$ ). On the other hand the MPPC can recharge through the large decoupling capacitor and the ground line.

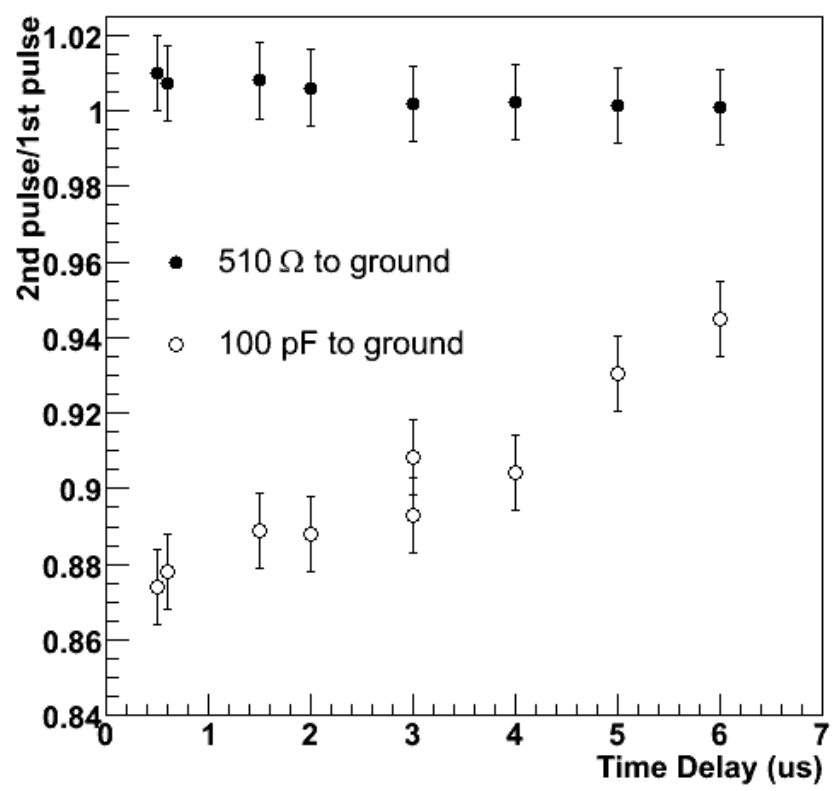

Figure 3. MPPC recovery time for two different readout circuit.

In order to investigate recovery further, a simple circuit was built. It is a simplified version of the schematics shown in Figure 1. The bias resistor was $100 \mathrm{k} \Omega$ and the decoupling capacitor was $100 \mathrm{nF}$. Only one branch was connected to the ASIC through a $10 \mathrm{pF}$ capacitor, without any resistor. The branch going to ground included either a $510 \Omega$ resistor or a $100 \mathrm{pF}$ capacitor. Figure 3 shows the height of the second laser pulse measured over the height of the first pulse measured by the ASIC. The first pulse fires about 100 MPPC pixels. Recovery is fast with the $510 \Omega$ resistor,which allows the MPPC to recharge through the $100 \mathrm{nF}$ capacitor, while using the $100 \mathrm{pF}$ capacitor essentially shuts off this branch and the recovery time constant is given by the $100 \mathrm{k} \Omega$ resistor in series with the power supply. The drawback of the fast recovery option is that the pulse shape at the input of the AFTER ASIC becomes bipolar, which can introduce a dead time for very large signals. Indeed, when the signal is very large the undershoot may drag the baseline well below the ADC dynamic range. 
Figure 1 shows the circuit chosen for the FGD, which is a compromise between a recovery time constant of 500 ns and a maximum deadtime due to the waveform undershoot of less than 500 ns. Using this circuit to couple the MPPC to the AFTER ASIC, the FGD specifications are met. Each photo-electron peak is well separated at low light level. The dynamic range goes from 1 to 400 pixel avalanches. The timing resolution is better than $5 \mathrm{~ns}$. Delayed pulse due to Michel positrons emitted by pion decay can be detected with a very good efficiency. The inefficiency incured by the waveform undershoot and the MPPC recovery time remains to be investigated in detail. We now investigate the MPPC behavior in order to define the MPPC running condition (bias voltage for a given temperature) and describe the FGD response in details.

\section{MPPC performances}

MPPCs can be characterized in terms of their gain, photon detection efficiency (PDE), dark noise, cross-talk and after-pulsing. The MPPC readout system used in the FGD can accomodate MPPC gains ranging from $5 \times 10^{4}$ to several milions. Thus, it is not the parameter that define the over-voltage. However, since the gain is linearly correlated with the overvoltage, it will be used as a monitor to ensure that the over-voltage of the FGD MPPCs are all within $10 \%$.

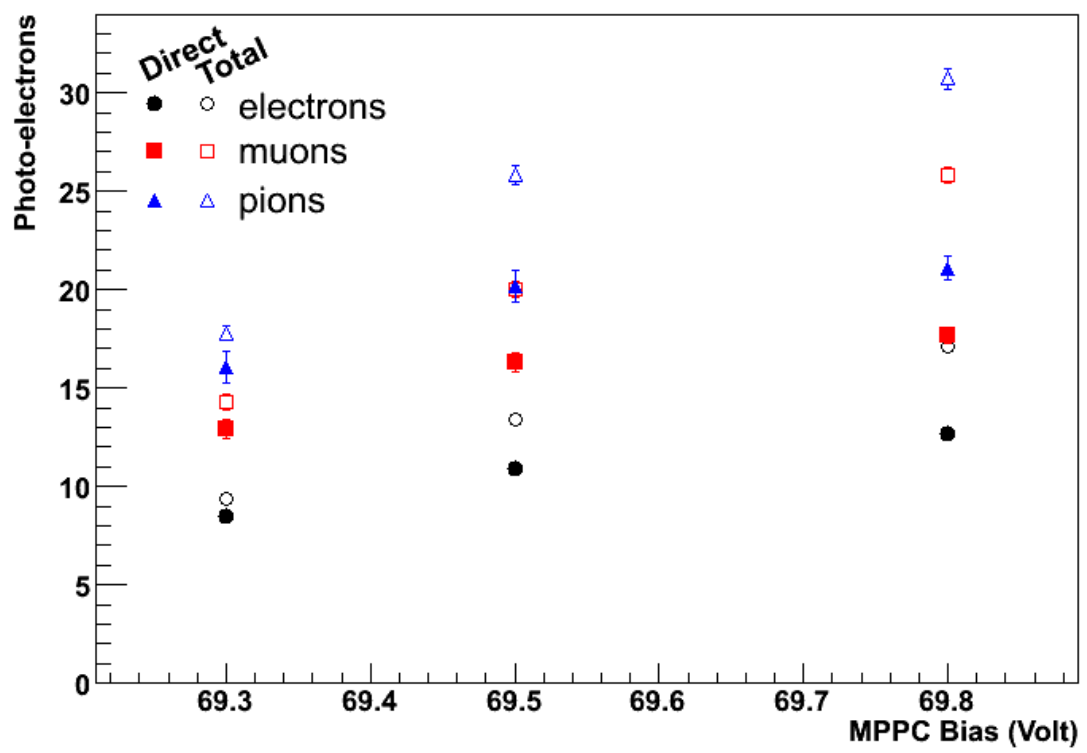

Figure 4. The total number of photo-electrons is the number of pixel avalanches, while the direct number is corrected forcross-talk and after-pulsing.

The PDE has been measured using a $120 \mathrm{MeV} / \mathrm{c}$ beam at TRIUMF. Figure 4 shows the most probable number of MPPC pixel avalanches and the infered number of photo-electrons for particles crossing FGD bars 2 meter away from the photo-sensor. The number of photoelectrons is infered by subtracting cross-talk and after-pulsing. The number of photo-electron measured for particles crossing the bar close to the photosensor is $50 \%$ larger. The wavelength shifting fibers had not been mirrored in this beam test. Separate tests show that mirroring increases the number of photo-electrons by $80-100 \%$ when particles cross the bar on the 
opposite side of the photosensor. At such beam momentum the electrons are minimum ionizing.Thus, the combination of scintillator, wavelength shifting fiber and MPPC provide the required minimum number of photo-electrons for minimum ionizing particles. It is important to notice that the PDE requirement is met at $69.5 \mathrm{~V}$ bias at $25^{\circ} \mathrm{C}$, which corresponds to an overvoltage of $0.9 \mathrm{~V}$.

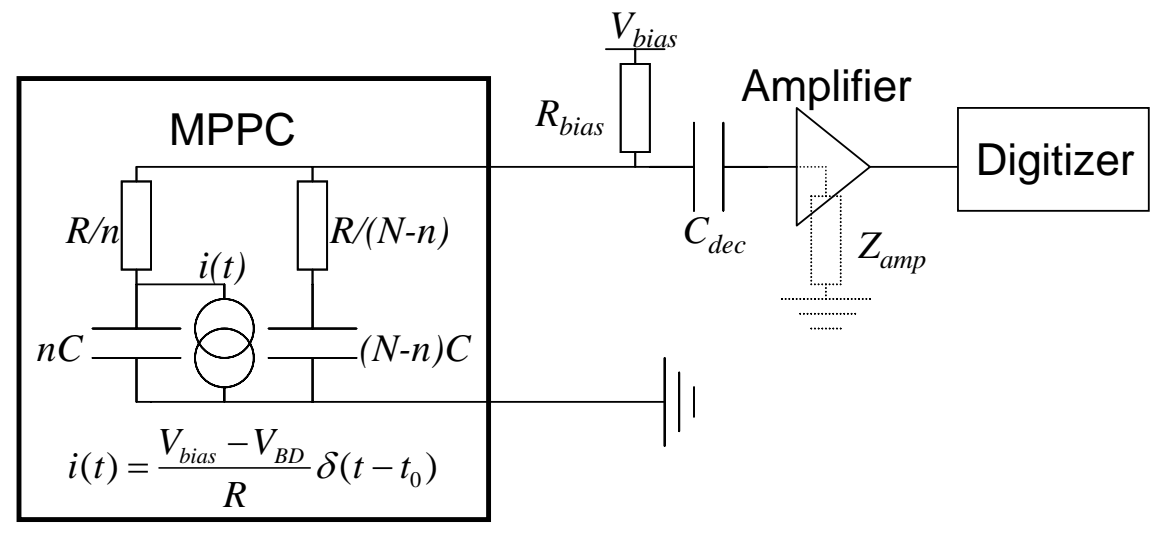

Figure 5. Setup used for the MPPC characterization. The equivalent circuit of a MPPC is also shown, with $R$ and $C$ the single pixel resistance and capacitance respecitively, $n$ the number of pixels firing at a time $t_{0}, N$ the total number of pixels and $V_{B D}$ the breakdown voltage. The avalanche is represented as a delta function because the rise time is significantly less than $1 \mathrm{~ns}$.

Indeed, it is advantageous to run at low over-voltage in order to keep the dark noise, crosstalk and after-pulsing low. Dark noise, cross-talk and after-pulsing have been studied using the setup shown schematically in Figure 5 . All the measurements have been performed at $25^{\circ} \mathrm{C}$, where the MPPC breakdown voltage was about $68.6 \mathrm{~V}$. The amplifier has an input impedance $\mathrm{Z}_{\text {amp }}$ of $50 \Omega$ and a $100 \mathrm{nF}$ decoupling capacitor ensures fast recovery of the MPPC bias. The amplifier input bandwidth is about $1 \mathrm{GHz}$ and the digitizer samples at $1 \mathrm{GHz}$. A trigger was generated by setting a 0.5 pixel avalanche trigger on the signal itself.

A pulse search is performed on the waveforms from the digitizer. The pulse finder is able to identify pulses separated by more than 3 ns. Figure 6 shows the distribution of the amplitude and time of the pulses when triggering on dark noise avalanches. The trigger pulse is found at time zero. Trigger pulse amplitudes corresponding to 2 or more avalanches are due to cross-talk. The pulses found after the trigger are due to dark noise and after-pulsing, which themselves may produce additional avalanches due to cross-talk. After-pulses are clearly visible between 5 and $30 \mathrm{~ns}$ after the trigger as their gain is lower than the nominal avalanche gain since the pixel bias is recovering with its typical RC constant of $8.75 \mathrm{~ns}$. In order to separate dark noise and afterpulsing, the timing distribution of the pulses following the trigger is projected, keeping only the first pulse immediately following the trigger. Using the distribution of first pulses ensures that chains of after-pulses (after-pulsing occuring several times on one pixel) do not bias the fit. This distribution is shown in Figure 7. The fit function is then the probability of having a dark noise pulse at $t$ and no after-pulse in $[0, t]$ plus the probability of having an after pulse at $t$ and no dark 
noise pulse in $[0, \mathrm{t}]: \quad d N / d t=\left(1-A p-A p \cdot e^{\frac{-t}{\tau}}\right) D N e^{-D N \cdot t}+e^{-D N \cdot t} \frac{A p}{\tau} e^{\frac{-t}{\tau}}$, with $D N$ the dark noise rate, $A p$ the probability that one pixel generates an after-pulse, and $\tau$ the after pulsing time constant. Using only one exponential does not give a good fit. On the other hand, the fit using two time constants with two different after-pulsing probabilities $\mathrm{Ap}_{\text {short }}$ and Aplong describes the data well. It has been suggested [3] that trapping should be described by 4 exponentials but the statistics available is too low to perform such fit.

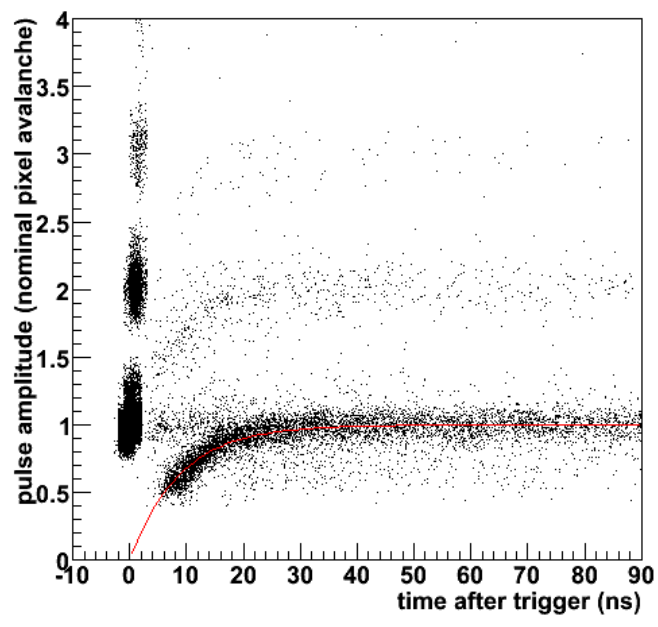

Figure 6. Distribution of the amplitude and time of the pulse found by the pulse finder at $70 \mathrm{~V}$. The red curve shows the expected 8.75 ns gain recovery.

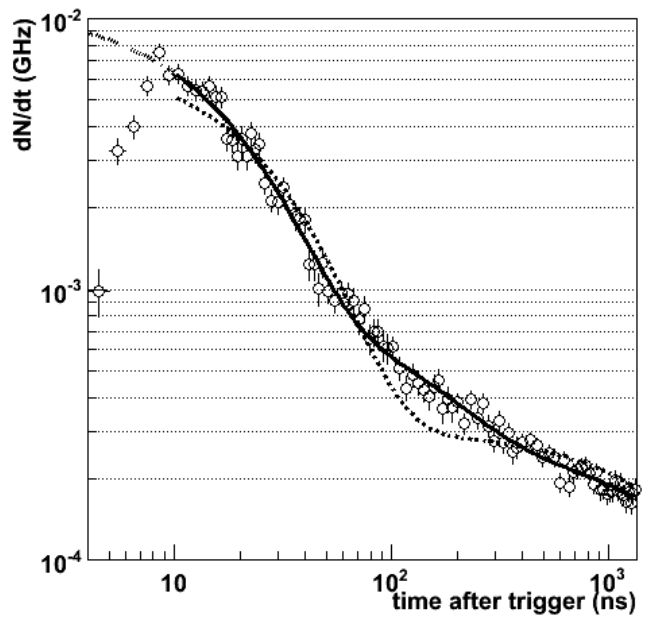

Figure 7. Time distribution of the pulse immediately following the trigger at 70V. Dash: one time constant fit. Solid: two time constant fit.

The time constants are shown in Figure 8 for different bias voltage. The parameters obtained from fitting the timing distribution of all the pulses are also shown. At $69.5 \mathrm{~V}$, the short time constant is $15 \pm 2$ (stat) \pm 2 (sys) ns and the long time constant is $112 \pm 21$ (stat) \pm 40 (sys) ns. The systematic errors are due to pulse finding inefficiencies, especially for pulses separated by less than $5 \mathrm{~ns}$, and to the limited range of the timing distribution. The time constant do not vary much with bias voltage. However, the long time constant is not well constrained because of its strong correlation with the dark noise rate, which can be disantengled with more statistics.

The after-pulsing probabilities are shown in Figure 9. They increase rapidly with bias voltage. At $69.5 \mathrm{~V}, \mathrm{Ap}_{\text {short }}=5.5 \pm 0.3$ (stat) \pm 0.6 (sys) $\%$, Ap long $=3.5 \pm 0.3$ (stat) \pm 0.6 (sys) $\%$. Crosstalk is found to be $5.5 \pm 0.1$ (stat) \pm 0.2 (sys) \% and the dark noise is $202 \pm 4$ (stat) \pm 20 (sys) kHz. The sum of both after-pulsing probabilities is shown in Figure 10 together with the cross-talk probability. At $70.2 \mathrm{~V}$, the total after-pulsing probability is larger than $25 \%$, which means the probability of having chains of after-pulses becomes significant. On the other hand, cross-talk remains below $10 \%$ even at $70.2 \mathrm{~V}$. 


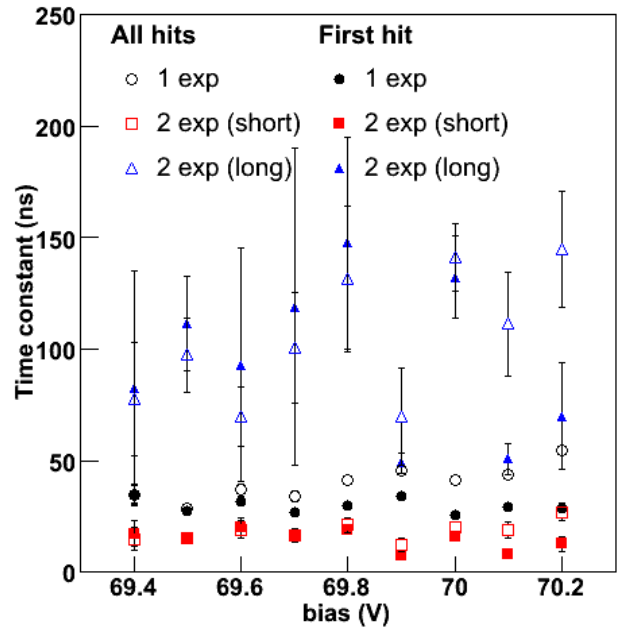

Figure 8.

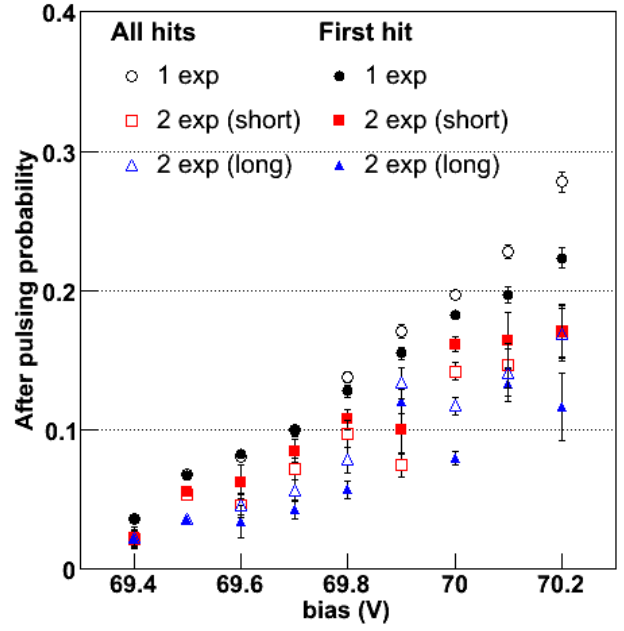

Figure 9

The first pulse analysis leads to lower dark noise than using all the hits because it does not include multiple pulses induced by after-pulsing. No saturation of the dark noise is seen. However, dark noise is not very well constrained in the first hit analysis because of its strong correlation with the long time constant. Indeed a dark noise of $0.4 \mathrm{MHz}$ corresponds to an equivalent time constant of $1 / 0.4 \mathrm{MHz}=250$ ns. As dark noise increases, its pattern becomes very similar to after-pulsing in the first hit analysis. In order to get a proper estimate of the dark noise, it must then be determined by other means. One strategy is described in [3]. It involves switching the bias voltage from just below the breakdown voltage (to empty the traps) to the desired bias voltage, and measuring the time distribution of the first dark noise pulse following the switch.

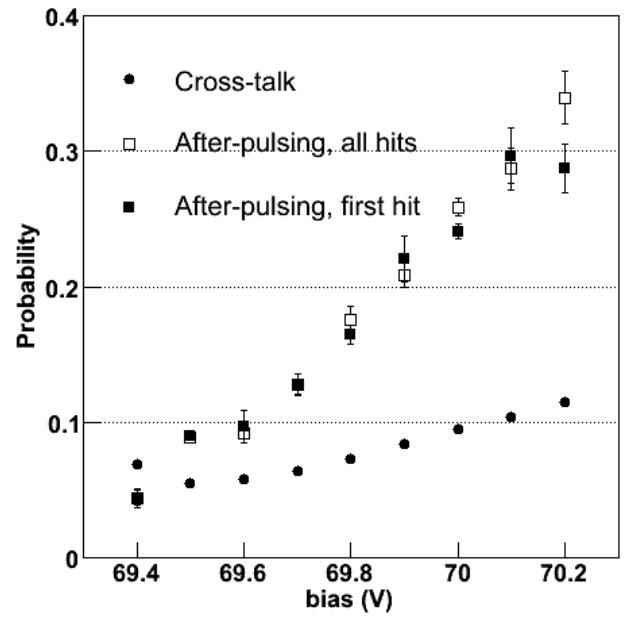

Figure 10

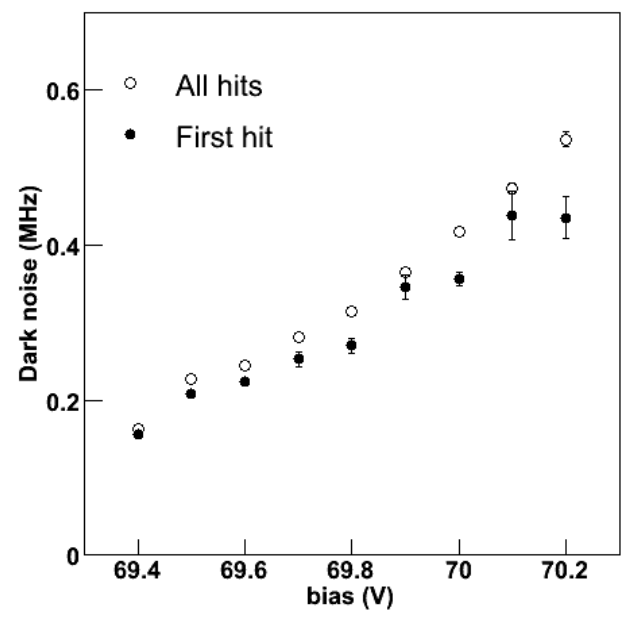

Figure 11 


\section{Conclusions}

The scintillation light from T2K Fine Grain Detector scintillator bars will be detected by 8448 MPPCs coupled to wavelength shifting fibers. The readout system relies on an ASIC designed for T2K Time Projection Chamber that includes a 120 ns rise time preamp-shaper and allows digitizing the signal at $50 \mathrm{MHz}$. The circuit coupling the MPPC to the AFTER ASIC is designed to accommodate the large MPPC dynamic range by splitting the signal in two attenuation branch and to provide a relatively short MPPC recovery time. This readout system meets the timing resolution requirements and provides a means of detecting delayed pulses from Michel positrons.

The MPPC performances match the FGD specifications. However, after-pulsing is large. Running at large over-voltage $(>1.5 \mathrm{~V})$ is not advisable because it leads to frequent chains of after-pulses. Fortunately, the MPPC gain and Photon Detection Efficiency meets the FGD specifications for over-voltages of the order of $1 \mathrm{~V}$, where the after-pulsing probability is on the order of $10 \%$. While it is established that MPPC performances are suitable for the FGD, estimating the after-pulsing and dark noise parameters precisely will require further tests and analyses.

\section{References}

[1] For information about T2K, see http://jnusrv01.kek.jp/public/t2k/

[2] Hamamatsu Photonics, MPPC catalogue, http://jp.hamamatsu.com/resources/products/ssd/pdf/mppc_kapd0002e02.pdf.

[3] S. Cova, A. Lacaita, G. Ripamonti, IEEE Trans. Electron Devices, vol 12, p 685, 1991 